\title{
A DECREASING PROPERTY OF SOLUTIONS OF PARABOLIC EQUATIONS WITH APPLICATIONS TO THERMOELASTICITY*
}

\section{BY}

W. A. DAY

Hertford College, Oxford

Introduction. The object of this paper is to establish a decreasing property of solutions of parabolic equations which satisfy boundary conditions of a somewhat unusual kind. We shall begin by illustrating, with the aid of two examples from the quasi-static theory of thermoelasticity, how such problems can arise.

Let us consider first the coupled partial differential equations

$$
\begin{gathered}
k \frac{\partial^{2} \theta}{\partial x^{2}}=c \frac{\partial \theta}{\partial t}+\theta_{0} \alpha(3 \lambda+2 \mu) \frac{\partial^{2} u}{\partial x \partial t} \\
(\lambda+2 \mu) \frac{\partial^{2} u}{\partial x^{2}}=\alpha(3 \lambda+2 \mu) \frac{\partial \theta}{\partial x}
\end{gathered}
$$

which describe the behavior of a slab $-l \leq x \leq l$ made of homogeneous and isotropic material. Here $\theta(x, t)$ is the temperature, $u(x, t)$ is the displacement component in the direction of the $x$-axis, $\theta_{0}$ is a uniform reference temperature, $k$ is the conductivity, $c$ is the specific heat at constant strain, $\alpha$ is the coefficient of expansion, and $\lambda, \mu$ are the elastic moduli. The reader is referred to Carlson's article [1] or to Boley and Weiner's treatise [2] for derivations of these equations.

We shall suppose the faces of the slab to be maintained at the reference temperature and to be clamped, that is to say

$$
\theta(-l, t)=\theta(l, t)=\theta_{0}, \quad u(-l, t)=u(l, t)=0 .
$$

We can write the differential equations as an energy equation

$$
k \frac{\partial^{2} \theta}{\partial x^{2}}=\theta_{0} \frac{\partial \eta}{\partial t}
$$

and a quasi-static equation of motion

$$
\partial \sigma / \partial x=0
$$

respectively, where

$$
\eta=\frac{c}{\theta_{0}}\left(\theta-\theta_{0}\right)+\alpha(3 \lambda+2 \mu) \frac{\partial u}{\partial x}
$$

* Received February 23, 1982. 
is the entropy, and

$$
\sigma=(\lambda+2 \mu) \frac{\partial u}{\partial x}-\alpha(3 \lambda+2 \mu)\left(\theta-\theta_{0}\right)
$$

is a stress component. We notice that

$$
\theta_{0} \frac{\partial^{2} \eta}{\partial x^{2}}=c \frac{\partial^{2} \theta}{\partial x^{2}}+\theta_{0} \alpha(3 \lambda+2 \eta) \frac{\partial^{3} u}{\partial x^{3}}=c^{*} \frac{\partial^{2} \theta}{\partial x^{2}},
$$

and, therefore, that the entropy is a solution of the heat equation

$$
k \frac{\partial^{2} \eta}{\partial x^{2}}=c^{*} \frac{\partial \eta}{\partial t}
$$

in which the constant

$$
c^{*}=c+\theta_{0} \alpha^{2} \frac{(3 \lambda+2 \mu)^{2}}{\lambda+2 \mu}
$$

is the specific heat at constant stress.

We notice too that the stress component is independent of $x$ and can be written as $\sigma(t)$. If now we express the entropy in the form

$$
\eta=\left(\frac{c(\lambda+2 \mu)}{\theta_{0} \alpha(3 \lambda+2 \mu)}+\alpha(3 \lambda+2 \mu)\right) \frac{\partial u}{\partial x}-\frac{c}{\theta_{0} \alpha(3 \lambda+2 \mu)} \sigma,
$$

and if we integrate with respect to $x$ and appeal to the fact that $u=0$ on the faces $x=-l$ and $x=l$, we see that

$$
\int_{-l}^{l} \eta(x, t) d x=-\frac{2 c l}{\theta_{0} \alpha(3 \lambda+2 \mu)} \sigma(t) .
$$

On the other hand, if we express the entropy in the form

$$
\eta=\frac{c^{*}}{\theta_{0}}\left(\theta-\theta_{0}\right)+\frac{\alpha(3 \lambda+2 \mu)}{\lambda+2 \mu} \sigma
$$

and appeal to the fact that $\theta=\theta_{0}$ on $x=-l$ and $x=l$, we see that

$$
\eta(-l, t)=\eta(l, t)=\frac{\alpha(3 \lambda+2 \mu)}{\lambda+2 \mu} \sigma(t)
$$

and, consequently, that the entropy satisfies the conditions

$$
\eta(-l, t)=\eta(l, t)=-\left(\frac{c^{*}-c}{c}\right) \cdot \frac{1}{2 l} \int_{-l}^{l} \eta(x, t) d x
$$

which connect the boundary values of the entropy with the average

$$
\frac{1}{2 l} \int_{-l}^{l} \eta(x, t) d x
$$

These conditions were derived previously in [3].

For our second example we consider the equations

$$
k \frac{\partial^{2} \theta}{\partial x^{2}}=c \frac{\partial \theta}{\partial t}+\theta_{0} B \frac{\partial^{3} u}{\partial x^{2} \partial t}, \quad A \frac{\partial^{4} u}{\partial x^{4}}=B \frac{\partial^{2} \theta}{\partial x^{2}}
$$


which describe the quasi-static flexure of a thermoelastic rod [4]. Here $\theta(x, t)$ is the temperature, $u(x, t)$ is the transverse displacement, the constant $A$ is the flexural rigidity, and the constant $B$ is a measure of the cross-coupling between thermal and mechanical effects. We shall suppose the ends $x=-l$ and $x=l$ to be maintained at the reference temperature $\theta_{0}$ and to be clamped, that is to say

$$
\begin{gathered}
\theta(-l, t)=\theta(l, t)=\theta_{0}, \\
u(-l, t)=\frac{\partial u}{\partial x}(-l, t)=u(l, t)=\frac{\partial u}{\partial x}(l, t)=0 .
\end{gathered}
$$

If we rewrite the equations as

$$
k \frac{\partial^{2} \theta}{\partial x^{2}}=\theta_{0} \frac{\partial \eta}{\partial t}, \quad \frac{\partial^{2} M}{\partial x^{2}}=0,
$$

respectively, where

$$
\eta=\frac{c}{\theta_{0}}\left(\theta-\theta_{0}\right)+B \frac{\partial^{2} u}{\partial x^{2}}
$$

is the entropy and

$$
M=A \frac{\partial^{2} u}{\partial x^{2}}-B\left(\theta-\theta_{0}\right)
$$

is the bending moment, we see that the entropy is a solution of the heat equation

$$
k \frac{\partial^{2} \eta}{\partial x^{2}}=\left(c+\theta_{0} \frac{B^{2}}{A}\right) \frac{\partial \eta}{\partial t}
$$

and that the bending moment has the form

$$
M(x, t)=\left(\frac{l-x}{2 l}\right) M(-l, t)+\left(\frac{l+x}{2 l}\right) M(l, t) .
$$

To deduce the boundary conditions on the entropy we use the equation

$$
\eta=\frac{1}{\theta_{0}}\left(c+\theta_{0} \frac{B^{2}}{A}\right)\left(\theta-\theta_{0}\right)+\frac{B}{A} M,
$$

which tells us that at the ends of the rod

$$
\eta(-l, t)=\frac{B}{A} M(-l, t), \quad \eta(l, t)=\frac{B}{A} M(l, t),
$$

and hence that the bending moment can be expressed in terms of the entropy as

$$
M(x, t)=\frac{A}{B}\left(\frac{l-x}{2 l}\right) \eta(-l, t)+\frac{A}{B}\left(\frac{l+x}{2 l}\right) \eta(l, t) .
$$

Next we appeal to the equation

$$
\left(B+\frac{c A}{\theta_{0} B}\right) \frac{\partial^{2} u}{\partial x^{2}}=\eta+\frac{c}{\theta_{0} B} M
$$


and to the identities

$$
\begin{aligned}
& (l-x) \frac{\partial^{2} u}{\partial x^{2}}=\frac{\partial}{\partial x}\left[(l-x) \frac{\partial u}{\partial x}+u\right], \\
& (l+x) \frac{\partial^{2} u}{\partial x^{2}}=\frac{\partial}{\partial x}\left[(l+x) \frac{\partial u}{\partial x}-u\right],
\end{aligned}
$$

which, since $u$ and $\partial u / \partial x$ vanish at $x=-l$ and $x=l$, imply that each of the integrals

$$
\begin{aligned}
& \int_{-l}^{l}(l-x)\left(\eta(x, t)+\frac{c}{\theta_{0} B} M(x, t)\right) d x, \\
& \int_{-l}^{l}(l+x)\left(\eta(x, t)+\frac{c}{\theta_{0} B} M(x, t)\right) d x
\end{aligned}
$$

must vanish. When we substitute for the bending moment we arrive, after some calculation, at the conclusion that the entropy satisfies the conditions

$$
\begin{aligned}
\eta(-l, t) & =-\frac{\theta_{0} B^{2}}{2 c A l^{2}} \int_{-l}^{l}(l-3 x) \eta(x, t) d x, \\
\eta(l, t) & =-\frac{\theta_{0} B^{2}}{2 c A l^{2}} \int_{-l}^{l}(l+3 x) \eta(x, t) d x
\end{aligned}
$$

which connect the boundary values of the entropy with two of its weighted averages.

Examples of this kind lead us to study the behavior of solutions of the heat equation, or of the more general parabolic equation

$$
\frac{\partial}{\partial x}\left(a(x) \frac{\partial \phi}{\partial x}\right)=b(x) \frac{\partial \phi}{\partial t}+c(x) \phi
$$

which satisfy the conditions

$$
\begin{aligned}
\phi(-l, t) & =\int_{-l}^{l} f(x) \phi(x, t) d x, \\
\phi(l, t) & =\int_{-l}^{l} g(x) \phi(x, t) d x,
\end{aligned}
$$

where $f(x)$ and $g(x)$ are known functions which we shall suppose to be continuous. The function $c(x)$ is not to be confused with the specific heat $c$ introduced previously.

If $f(x)$ and $g(x)$ vanish identically the boundary conditions collapse to the conditions

$$
\phi(-l, t)=\phi(l, t)=0,
$$

and it is known that provided $a(x)$ and $b(x)$ are positive and $c(x)$ is non-negative the maximum modulus

$$
\max _{-l \leqslant x \leqslant l}|\phi(x, t)|
$$

is a decreasing function of $t$, in the wide sense, whatever the initial values taken by $\phi(x, t)$ on $t=0$. 
In the case of the heat equation, this result is due to Pólya and Szegö [5]. A different method of proof, which can easily be extended to include general types of parabolic equations, was discovered by Bellman [6]. Bellman's method, with appropriate modifications, suffices for our purposes.

We should like to prove that the maximum modulus of $\phi(x, t)$ decreases even when $f(x)$ and $g(x)$ do not vanish identically, for then we should have proved that the maximum modulus of the entropy must decrease in each of our examples from thermoelasticity. However, no such result can be true without some restrictions upon $f(x)$ and $g(x)$, as we can see by contructing specific examples or, more instructively, with the aid of the following considerations which suggest what the appropriate restrictions might be.

For the moment let us confine our attention to the heat equation

$$
\partial^{2} \phi / \partial x^{2}=\partial \phi / \partial t
$$

and let $D(\omega)$ be the $2 \times 2$ determinant

$$
\left|\begin{array}{cc}
-\sinh \omega l-\int_{-l}^{l} f(x) \sinh \omega x d x & \cosh \omega l-\int_{-l}^{l} f(x) \cosh \omega x d x \\
\sinh \omega l-\int_{-l}^{l} g(x) \sinh \omega x d x & \cosh \omega l-\int_{-l}^{l} g(x) \cosh \omega x d x
\end{array}\right| .
$$

If, for some real $\omega$ which is different from zero, $D(\omega)$ vanishes, there are numbers $\delta$ and $\varepsilon$, not both zero, such that

$$
\begin{gathered}
-\delta \sinh \omega l+\varepsilon \cosh \omega l=\delta \int_{-l}^{l} f(x) \sinh \omega x d x+\varepsilon \int_{-l}^{l} f(x) \cosh \omega x d x, \\
\delta \sinh \omega l+\varepsilon \cosh \omega l=\delta \int_{-l}^{l} g(x) \sinh \omega x d x+\varepsilon \int_{-l}^{l} g(x) \cosh \omega x d x,
\end{gathered}
$$

and consequently the function

$$
\phi(x, t)=(\delta \sinh \omega x+\varepsilon \cosh \omega x) \exp \omega^{2} t
$$

is a solution of the heat equation which satisfies each of the boundary conditions; its maximum modulus, though, is an exponentially increasing function of $t$. Thus the desired conclusion cannot hold if $f(x)$ and $g(x)$ are such that $D(\omega)$ vanishes at a value of $\omega$ other than $\omega=0$.

Since the determinant depends continuously upon $\omega$ and has the properties

$$
\begin{aligned}
\frac{d}{d \omega} D(0)= & -\left(l+\int_{-l}^{l} x f(x) d x\right)\left(1-\int_{-l}^{l} x g(x) d x\right) \\
& -\left(1-\int_{-l}^{l} f(x) d x\right)\left(l-\int_{-l}^{l} x g(x) d x\right), \\
D(\omega) \sim & -2 \cosh \omega l \sinh \omega l \quad \text { as } \omega \rightarrow \infty,
\end{aligned}
$$


it is clear that it must vanish at a non-zero value of $\omega$ if

$$
\left(l+\int_{-l}^{l} x f(x) d x\right)\left(1-\int_{-l}^{l} g(x) d x\right)+\left(1-\int_{-l}^{l} f(x) d x\right)\left(l-\int_{-l}^{l} x g(x) d x\right)<0
$$

and in that case the desired conclusion cannot hold.

On the other hand, if $f(x)$ and $g(x)$ satisfy the conditions

$$
\int_{-l}^{l}|f(x)| d x<1, \quad \int_{-l}^{l}|g(x)| d x<1,
$$

and if $\omega$ is non-zero, the upper left-hand entry in the determinant is negative, each of the three remaining entries is positive, and the determinant is negative. Thus the conditions imposed upon $f(x)$ and $g(x)$ ensure that $D(\omega)$ vanishes only at $\omega=0$. We shall prove that, in fact, these conditions are sufficient to ensure that the maximum modulus decreases not just for solutions of the heat equation but for solutions of more general parabolic equations as well.

The decreasing property. In precise terms our result is: Let $\phi(x, t)$ be a solution of the parabolic equation

$$
\frac{\partial}{\partial x}\left(a(x) \frac{\partial \phi}{\partial x}\right)=b(x) \frac{\partial \phi}{\partial t}+c(x) \phi
$$

which satisfies the boundary conditions

$$
\begin{gathered}
\phi(-l, t)=\int_{-l}^{l} f(x) \phi(x, t) d x, \\
\phi(l, t)=\int_{-l}^{l} g(x) \phi(x, t) d x
\end{gathered}
$$

for $t>0$. Let $a(x), b(x), c(x), f(x)$, and $g(x)$ be continuous, let $a(x)$ and $b(x)$ be positive, let $c(x)$ be non-negative, and let $f(x)$ and $g(x)$ satisfy

$$
\int_{-l}^{l}|f(x)| d x<1, \quad \int_{-l}^{l}|g(x)| d x<1 .
$$

Then the maximum modulus

$$
\max _{-l \leqslant x \leqslant l}|\phi(x, t)|
$$

is a decreasing function of $t$ for $t>0$.

This result has the following implications for our two examples from thermoelasticity: The maximum modulus of the entropy decreases in the first example if

$$
\frac{c^{*}-c}{c}<1
$$

that is if

$$
\theta_{0} \alpha^{2}(3 \lambda+2 \mu)^{2}<(\lambda+2 \mu) c
$$


and in the second example if

$$
5 \theta_{0} B^{2}<3 A c \text {. }
$$

The latter of these implications appears to be new; the former is known [3].

The key to establishing the monotone property is to consider a sequence of functions

$$
I_{n}(t)=\int_{-l}^{l} p(x) b(x) \phi(x, t)^{2 n} d x \quad(n=1,2,3, \ldots),
$$

where $p(x)$ is a weighting function which is strictly positive for $-l<x<l$. A standard argument, which Beckenbach and Bellman [7, chapter 4, section 24] attribute to $M$. Riesz, establishes that

$$
I_{n}(t)^{1 / 2 n} \rightarrow \max _{-l \leqslant x \leqslant l}|\phi(x, t)| \quad \text { as } n \rightarrow \infty
$$

and, thus, in order to prove the theorem it is enough to show that $I_{n}(t)$ is a decreasing function of $t$, at least for all sufficiently large $n$.

We choose $p(x)$ to be the solution of the boundary-value problem

$$
\frac{d}{d x}\left(a(x) \frac{d p}{d x}\right)=1, \quad p(-l)=p(l)=0,
$$

that is, $p(x)$ is the function

$$
\left(\int_{-l}^{x} \frac{1}{a(y)} d y \int_{x}^{l} \frac{(y-x)}{a(y)} d y+\int_{x}^{l} \frac{1}{a(y)} d y \int_{-l}^{x} \frac{(x-y)}{a(y)} d y\right) / \int_{-l}^{l} \frac{1}{a(y)} d y .
$$

We see that $p(x)$ is positive for $-l<x<l$, as required.

Once we have chosen $p(x)$ in this way, the identity

$$
\begin{aligned}
2 n p \frac{\partial}{\partial x}\left(a \frac{\partial \phi}{\partial x}\right) \phi^{2 n-1}= & \frac{\partial}{\partial x}\left(2 n p a \frac{\partial \phi}{\partial x} \phi^{2 n-1}-a \frac{d p}{d x} \phi^{2 n}\right) \\
& +\frac{d}{d x}\left(a \frac{d p}{d x}\right) \phi^{2 n}-2 n(2 n-1) p a \phi^{2 n-2}\left(\frac{\partial \phi}{\partial x}\right)^{2}
\end{aligned}
$$

leads us to conclude that

$$
2 n p \frac{\partial}{\partial x}\left(a \frac{\partial \phi}{\partial x}\right) \phi^{2 n-1} \leq \frac{\partial}{\partial x}\left(2 n p a \frac{\partial \phi}{\partial x} \phi^{2 n-1}-a \frac{d p}{d x} \phi^{2 n}\right)-\phi^{2 n},
$$

and we deduce that the derivative

$$
\begin{aligned}
\frac{d I_{n}}{d t}= & \int_{-l}^{l} 2 n p b \phi^{2 n-1} \frac{\partial \phi}{\partial t} d x \\
= & \int_{-l}^{l}\left(2 n p \frac{\partial}{\partial x}\left(a \frac{\partial \phi}{\partial x}\right) \phi^{2 n-1}-2 n p c \phi^{2 n}\right) d x \\
& \leq \int_{-l}^{l} 2 n p \frac{\partial}{\partial x}\left(a \frac{\partial \phi}{\partial x}\right) \phi^{2 n-1} d x \\
& \leq \int_{-l}^{l}\left(\frac{\partial}{\partial x}\left(2 n p a \frac{\partial \phi}{\partial x} \phi^{2 n-1}-a \frac{d p}{d x} \phi^{2 n}\right)-\phi^{2 n}\right) d x
\end{aligned}
$$




$$
\begin{aligned}
& =a(-l) \frac{d p}{d x}(-l) \phi(-l, t)^{2 n}-a(l) \frac{d p}{d x}(l) \phi(l, t)^{2 n}-\int_{-l}^{l} \phi(x, t)^{2 n} d x \\
& =a(-l) \frac{d p}{d x}(-l)\left(\int_{-l}^{l} f \phi d x\right)^{2 n}-a(l) \frac{d p}{d x}(l)\left(\int_{-l}^{l} g \phi d x\right)^{2 n}-\int_{-l}^{l} \phi^{2 n} d x .
\end{aligned}
$$

If now we estimate the first and second terms on the right-hand side with the aid of Hölder's inequality and note that $d p / d x$ is positive at $x=-l$ and negative at $x=l$ we arrive at the inequality

$$
\frac{d I_{n}}{d t} \leq c_{n} \int_{-l}^{l} \phi^{2 n} d x
$$

in which the coefficient $c_{n}$ equals

$$
\begin{aligned}
& -1+a(-l) \frac{d p}{d x}(-l)\left(\int_{-l}^{l}|f|^{2 n /(2 n-1)} d x\right)^{2 n-1} \\
& \qquad-a(l) \frac{d p}{d x}(l)\left(\int_{-l}^{l}|g|^{2 n /(2 n-1)} d x\right)^{2 n-1}
\end{aligned}
$$

As $n$ tends to infinity,

$$
\begin{aligned}
& \int_{-l}^{l}|f|^{2 n /(2 n-1)} d x \rightarrow \int_{-l}^{l}|f| d x<1, \\
& \int_{-l}^{l}|g|^{2 n /(2 n-1)} d x \rightarrow \int_{-l}^{l}|g| d x<1,
\end{aligned}
$$

and therefore $c_{n}$ tends to -1 . In particular, we must have $c_{n} \leq-\frac{1}{2}$ for all large $n$ and

$$
\frac{d I_{n}}{d t} \leq-\frac{1}{2} \int_{-l}^{l} \phi^{2 n} d x \leq 0 .
$$

Thus $I_{n}(t)$ is a decreasing function of $t$ for all large $n$ and the proof is complete.

\section{REFERENCES}

[1] D. E. Carlson, Linear thermoelasticity, in Encyclopedia of physics, Vol. VIa/2, Springer, Berlin (1972)

[2] B. A. Boley and J. H. Weiner, Theory of thermal stresses, Wiley, New York (1960)

[3] W. A. Day, Extensions of a property of the heat equation to linear thermoelasticity and other theories, Quart. Appl. Math. (to appear)

[4] W. A. Day, The flexure of a thermoelastic rod, to be published

[5] G. Pólya and G. Szegö, Sur quelques propriétés qualitatives de la propagation de la chaleur, C. R. Acad. Sci. (Paris) 192, 1340-1342 (1931)

[6] R. Bellman, A property of summation kernels, Duke Math J. 15, 1013-1019 (1948)

[7] E. F. Beckenbach and R. Bellman, Inequalities, Springer, Berlin (1965) 